\title{
DOS NUEVOS TAXA DE BURSERA (BURSERACEAE) DE BAJA CALIFORNIA SUR, MÉXICO
}

\author{
José Luis León de La LuZ y José JuAn Pérez-Navarro
}

Centro de Investigaciones Biológicas del Noroeste,SC, Herbario HCIB, Apdo. postal 128, 23000 La Paz, Baja California Sur, México. jlleon04@cibnor.mx

\section{RESUMEN}

Se describen e ilustran dos nuevos taxa del género Bursera de la región de Los Cabos, en el extremo meridional de la Península de Baja California, México. Uno de estos es designado como B. littoralis y el otro como B. rupicola. El primero tiene cierta similitud con B. filicifolia y B. laxiflora del sur de la península e incluso con B. ribana del occidente del país, pero difiere de éstas por el menor tamaño de las hojas y folíolos, tiene también un porte arbustivo con ramificación compacta e intrincada, y particularmente porque habita en la duna costera. El segundo taxon se relaciona con B. epinnata del sur de la península, distinguiéndose de ésta por presentar hojas casi siempre unifolioladas con folíolos membranáceos, brillantes, las inflorescencias cortas con menor número de flores, y particularmente por su hábito arbustivo de ramas colgantes. Ambas especies pertenecen a la sección Bullockia por tener el ovario 2-locular, fruto 2-valvado, catafilos manifiestos, flores 4(5)-meras y corteza no exfoliante.

Palabras clave: Baja California, Bursera, México, región de Los Cabos, taxonomía.

\begin{abstract}
We describe and illustrate two new taxa of Bursera from the Cape Region in the southern sector of the Baja California Peninsula, Mexico. One is named B. littoralis and the other B. rupicola. The former is morphologically related to B. filicifolia and B. laxiflora of the southern peninsula and still with B. ribana of western Mexico, but differs in its smaller leaves and leaflets, the shrubby habit with dense and intricate branching, and in its habitat on the coastal dunes. The second taxon is related to B. epinnata of the southern peninsula, differing from it in its almost always unifoliolate, shiny, and membranaceous
\end{abstract}


leaves with scattered simple and glandular trichomes, short inflorescences with few flowers and particularly in its shrubby growth habit with pendent branches. Both species belong to the Bullockia section, having a bilocular ovary, bivalvate fruit, well developed cataphylls, 4(5)-merous flowers, and non-exfoliating bark.

Key words: Baja California, Bursera, Cape region, Mexico, taxonomy.

El género Bursera Jacquin ex L. (Burseraceae) se encuentra diversificado en las regiones tropicales y subtropicales mexicanas; una recopilación reciente del género registra 82 taxa para México (Rzedowski et al., 2005). Entre las principales determinantes ambientales señaladas por Rzedowski y Kruse (1979) y Johnson (1992) está el hecho de su exclusión de regiones con incidencia regular de temperaturas por debajo del punto de congelación. El género comprende árboles y arbustos semi-suculentos de uno a más de 30 metros de alto, de hojas alternas, simples a bi-pinnadas, deciduas la mayor parte del año, variables en tamaño y forma aun en la misma planta, la floración aparece generalmente al inicio de la época de lluvias; la mayor parte de las especies son dioicas y otras polígamo-dioicas. Los representantes del género son generalistas en cuanto a polinizadores, mientras que sus frutos son dispersados por aves. Se trata de un grupo diversificado y exitoso dentro de la comunidad del bosque tropical caducifolio mexicano (Rzedowski y Kruse, 1979).

No obstante la publicación de dos obras monumentales sobre la flora de la Península de Baja California (Shreve y Wiggins, 1964 y Wiggins, 1980) y de varias monografías taxonómicas, todavía ciertas áreas de la comarca se encuentran sin exploración botánica, particularmente dentro de las regiones montañosas.

La región de Los Cabos, ubicada en la porción sur de la península, es el asiento de una importante proporción de endemismos de vegetales con flores en los diferentes sectores que la componen (León de la Luz et al., 1999). Kohlmann y Sánchez-Colón (1984) al analizar la distribución geográfica de las especies mexicanas de Bursera, concluyeron que esta franja es un área de concentración de especies endémicas, conclusión ratificada en la recopilación de Rzedowski et al. (2005) sobre el mismo género.

En este trabajo se presentan dos componentes nuevos de Bursera, producto de la exploración botánica de los autores. Bursera littoralis se encuentra en el ambiente costero, entre los límites de la región de Los Cabos y la subprovincia florística de Los Llanos de Bahía Magdalena (Shreve y Wiggins, 1964); crece en la vertiente protegida de la duna. La segunda especie, Bursera rupicola, se ubica francamente 
dentro de la región de Los Cabos, en una comunidad fisonómicamente similar al bosque tropical caducifolio, en un ambiente muy particular, pues prospera en una cañada honda y estrecha en la cual el factor luz es determinante para la existencia de muchos vegetales, situación que ha permitido en ese mismo sitio el desarrollo de otros endemismos de la flora peninsular. La descripción de los dos taxa nuevos se detalla a continuación.

Bursera littoralis León de la Luz et Pérez Navarro, sp. nov. Fig. 1.

Frutex demissa, dioica, resina aromatica. Caulis cortice grisea non exfolianti; cataphylla inconspicua. Folia imparipinnata in apice brachyblastorum plerumque conglomerata, petiolata, rhachidi alata margine integro; foliola 7-11, petiolulata vel sessilia, petiolulis alatis, foliolis ovatis, ad apicem basemque rotundatis, margine profunde crenato, leviter revoluto, pubescentia dispersa, in nervis magis abundanti. Inflorescentiae in paniculis brevibus, laxis, pedunculatis, ad 6 floribus, aut monocasiis aut dichasiis vel floribus solitariis pedicellatis, pubescentibus; bracteolis filiformibus, pilosis. Flores masculi 4-meri; calyx lobulis lanceolatis, supra subtusque dense pilosis; petalis incurvatis; stamina 8. Flores feminei 4-meri; calyx lobulis lanceolatis, supra subtusque pilosis; petala incurvata, mucronata; ovarium 2-loculare, glabrum, stigma 2-lobato. Fructus solitarii vel aggregati, 2-valvati, putamine nigro, ad 2/3 longitudinis pseudoarillo aurantiaco vel rubro obtecto.

Arbustos hasta $1.5 \mathrm{~m}$ de alto, dioicos, con resina aromática; tronco con corteza gris no exfoliante, ramas jóvenes grisáceo-glaucas a pardo-rojizas, pubescentes a glabrescentes, ramas maduras glabras; catafilos deltoides, de 0.8 a $1.2 \mathrm{~mm}$ de largo por 1 a $1.5 \mathrm{~mm}$ de ancho en la base, pubescentes en la superficie exterior, glabros en la interior; hojas pinnadas o a veces bipinnadas, con 7 a 11 folíolos, generalmente aglomeradas en el ápice de los braquiblastos, pecíolos de 4 a $8 \mathrm{~mm}$ de largo, pubescentes y ligeramente ensanchados, láminas deltoides a lanceoladas en contorno general, 2.5 a $5 \mathrm{~cm}$ de largo por 1.2 a $1.5 \mathrm{~cm}$ de ancho, pubescentes a glabrescentes con la edad; raquis alado, ala angosta de $0.4 \mathrm{~mm}$ de ancho por lado, con nervadura central evidente, margen entero, revoluto, con tricomas esparcidos, más abundantes en los entrenudos, formando pequeños mechones; folíolos peciolulados o sésiles, ovados a orbiculares, ápice y base redondeadas, membranáceos, margen profundamente crenado, 2 a 6 lobos por lado, ciliado, haz brillante con tricomas esparcidos, simples, curvados en el ápice, más abundantes en la nervadura primaria y tricomas glandulares diminutos, envés piloso principalmente en la nervadura 


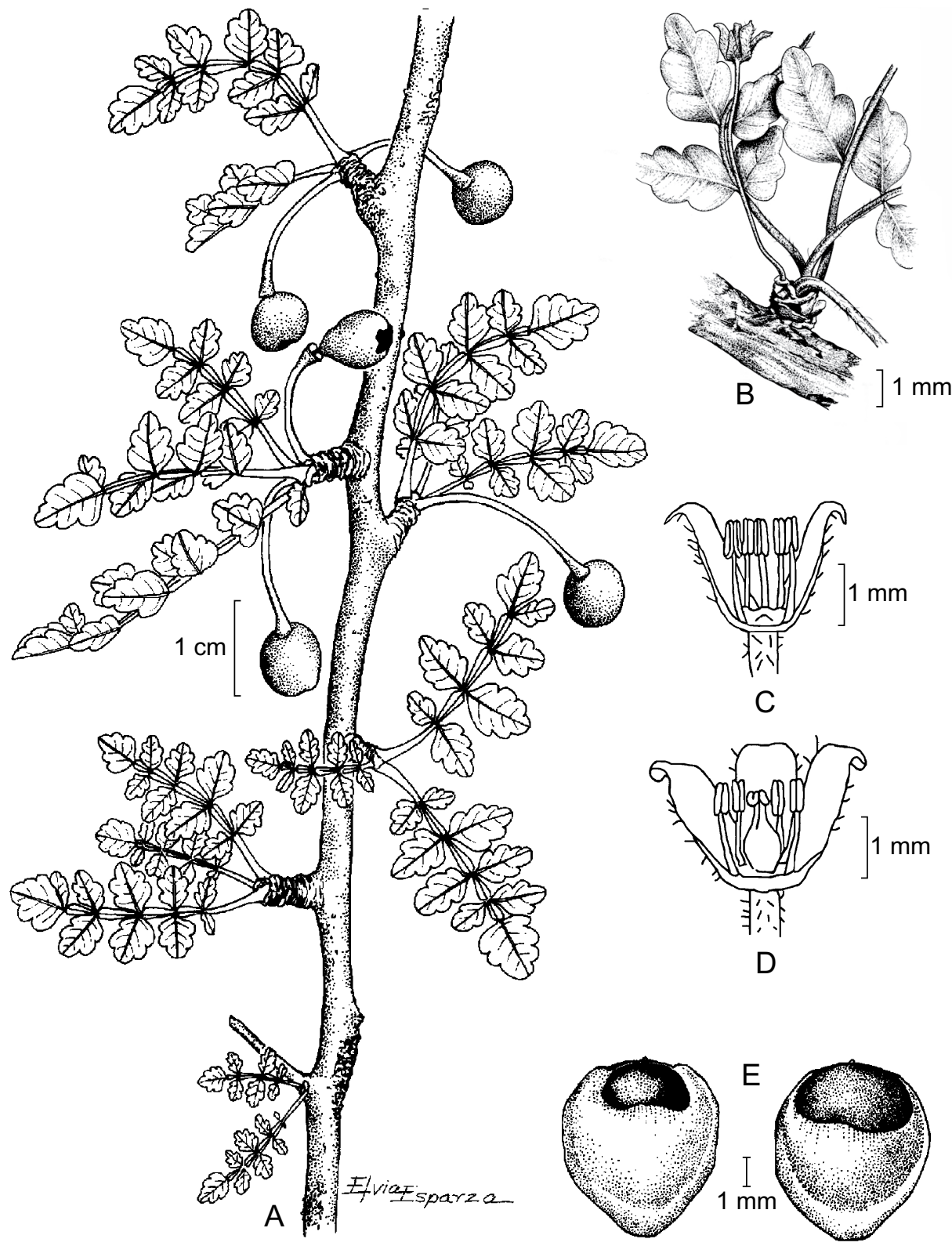

Fig. 1. Bursera littoralis León de la Luz y Pérez Navarro. A. Rama con hojas y frutos; B. Detalle de la inflorescencia femenina con flor solitaria; C. Flor masculina, desprovista de varios segmentos; D. Flor femenina, desprovista de varios segmentos; E. Hueso, visto por ambas superficies. Ilustrado por Elvia Esparza y Oscar Armendariz. 
primaria y en las secundarias, nervaduras rojizas en ambas superficies, peciólulos ensanchados hasta de $1 \mathrm{~mm}$ de largo, con pubescencia esparcida; inflorescencias en panículas cortas, laxas, pedunculadas, pedúnculos hasta de $25 \mathrm{~mm}$ de largo, con tricomas simples y tricomas glandulares, flores hasta 6 , pediceladas, agrupadas en monocasios, dicasios o bien solitarias, pedicelos de 5 a $13 \mathrm{~mm}$ de largo, con tricomas simples y tricomas glandulares, bractéolas lineares, $0.5 \mathrm{~mm}$ de largo, pilosas; flores masculinas 4-meras, cáliz con lóbulos lanceolados, de 1.5 a $2.5 \mathrm{~mm}$ de largo y $0.4 \mathrm{~mm}$ ancho, densamente pilosos en ambas superficies, pétalos reflexos en la antesis, blanquecino-amarillentos, oblongo-lanceolados, de $2 \mathrm{~mm}$ de largo y 0.8 a 1 $\mathrm{mm}$ de ancho, ápice recurvado, estambres 8 , filamentos 1.2 a $1.6 \mathrm{~mm}$ de largo, anteras oblongas, 0.5 a $0.6 \mathrm{~mm}$ de largo, gineceo vestigial evidente, disco nectarífero 8-lobulado, de menos de $1 \mathrm{~mm}$ de diámetro; flores femeninas 4-meras, cáliz con lóbulos lanceolados, de $2 \mathrm{~mm}$ de largo y $1.2 \mathrm{~mm}$ de ancho, pilosos en ambas superficies, pétalos oblongo-lanceolados, de $2 \mathrm{~mm}$ de largo, ápice con papilas, recurvado, mucronado, superficie exterior pilosa, interior glabra, estaminodios con filamentos de $0.5 \mathrm{~mm}$ de largo, anteras de $0.3 \mathrm{~mm}$ de largo, disco nectarífero de 1 a $1.2 \mathrm{~mm}$ de diámetro, ovario bilocular, 0.8 a $1 \mathrm{~mm}$ de largo, glabro, estigma bilobado, 0.1 $\mathrm{mm}$ de largo, $0.2 \mathrm{~mm}$ de grosor; pedúnculo fructífero arqueado, drupas solitarias o agrupadas, bivalvadas, esferoides, marcadamente apiculadas, de 6 a 8(-9) $\mathrm{mm}$ de largo, por 6 a $8 \mathrm{~mm}$ de ancho, endocarpio negro, cubiertas en $2 / 3$ de su largo por un pseudo-arilo anaranjado o rojo.

Tipo: México, Baja California Sur, municipio La Paz: $1.5 \mathrm{~km}$ oeste del ejido Melitón Albáñez, 19 junio 2000, J.J. Pérez Navarro 1246 (holotipo: HCIB; isotipo: MEXU).

Material adicional examinado: Baja California Sur. Municipio: La Paz: gravelly slope 2.8 miles south of Todos Santos along road to Cabo San Lucas, I.L. Wiggins 14519 (MEXU); región de Todos Santos, G. Guzmán Huerta H-76 (ENCB); 1.5 km oeste del ejido Melitón Albáñez, J.J. Pérez Navarro 1260 (HCIB), 1261 (HCIB); huerta El Tecolote, ca. las Playitas de Todos Santos, J.J. Pérez Navarro 1277 (HCIB); playa al este del estero de San José, J.J. Pérez Navarro 1290 (HCIB); predio Agua Blanca, aproximadamente $3.5 \mathrm{~km}$ sureste del ejido Melitón Albáñez, J.J. Pérez Navarro 1342 (HCIB); cauce del arroyo ca. las Playitas de Todos Santos, J.J. Pérez Navarro 1344 (HCIB); $1344 b$ (HCIB); Cabo San Lucas, J.J. Pérez Navarro 1345 (HCIB); playa del poblado Melitón Albáñez, J.L. León de la Luz y R. Medina Lemos 08-140 (HCIB, MEXU). 
Bursera littoralis constituye un elemento endémico del margen noroccidental de los límites de la región del Cabo y las planicies de bahía Magdalena, en el litoral del Pacífico de Baja California Sur (Fig. 2), cuya población más amplia se ubica en las dunas de la zona costera al norte del Trópico de Cáncer, pero se registran también, aunque como individuos solitarios, en comunidades de matorral sarcocaule en la misma vertiente del Pacífico (Cabo San Lucas y San José del Cabo), ocasionalmente compartiendo hábitat con otras especies del género, como $B$. odorata Brandegee, B. epinnata (Rose) Engl. y B. microphylla A. Gray. Este taxon desarrolla sus hojas, florece y fructifica durante la temporada de lluvias (variable entre los meses de julio y septiembre).

Sin duda, la afinidad morfológica más cercana es con B. filicifolia Brandegee, de la cual se distingue por presentar las hojas, pecíolos y sépalos con pubescencia esparcida a glabrescentes, las flores en menor número y la inflorescencia más com-

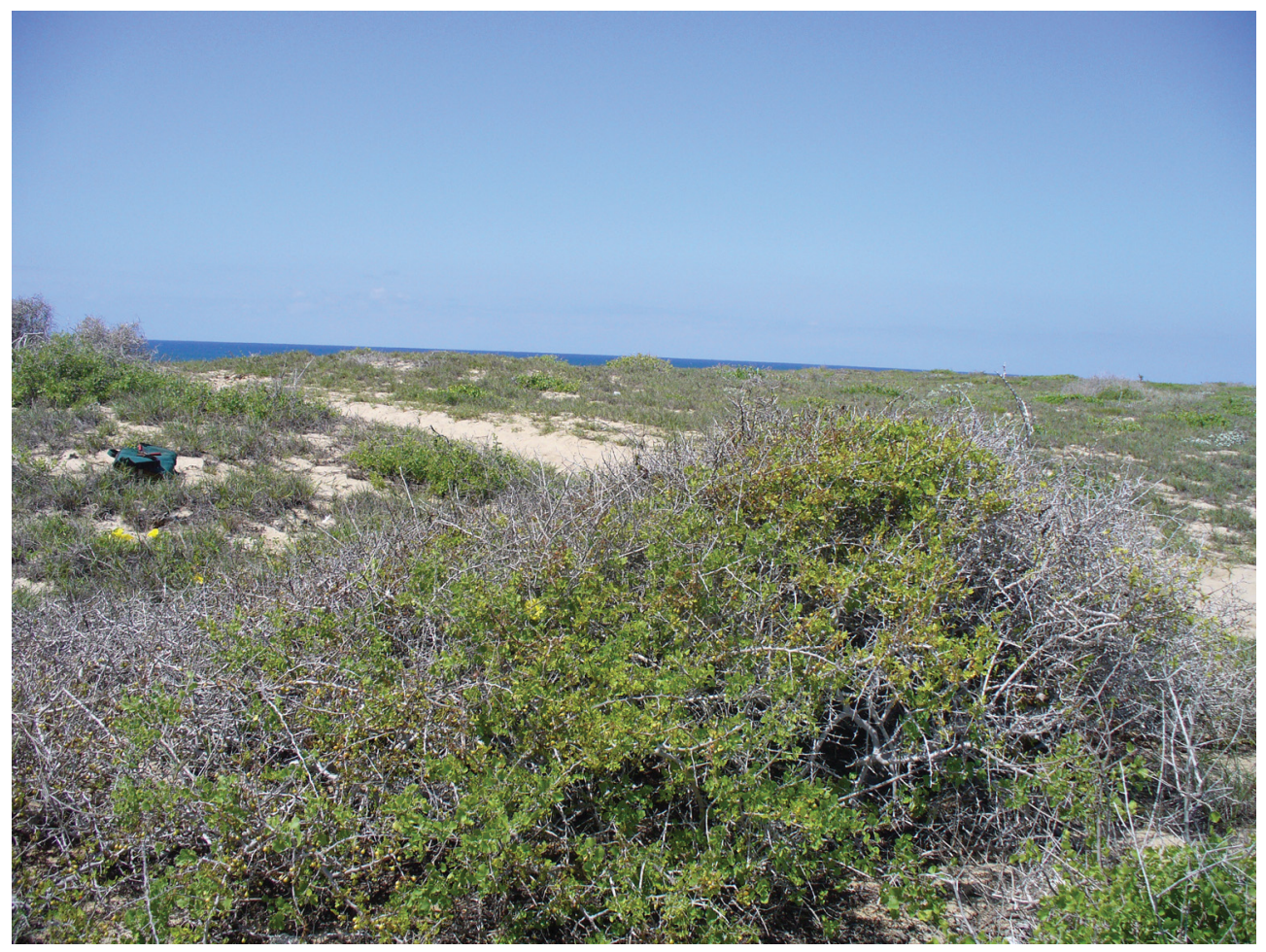

Fig. 2. Ejemplar de B. littoralis en su habitat típico. 
León de la Luz y Pérez-Navarro: Dos nuevos taxa de Bursera de Baja California Sur

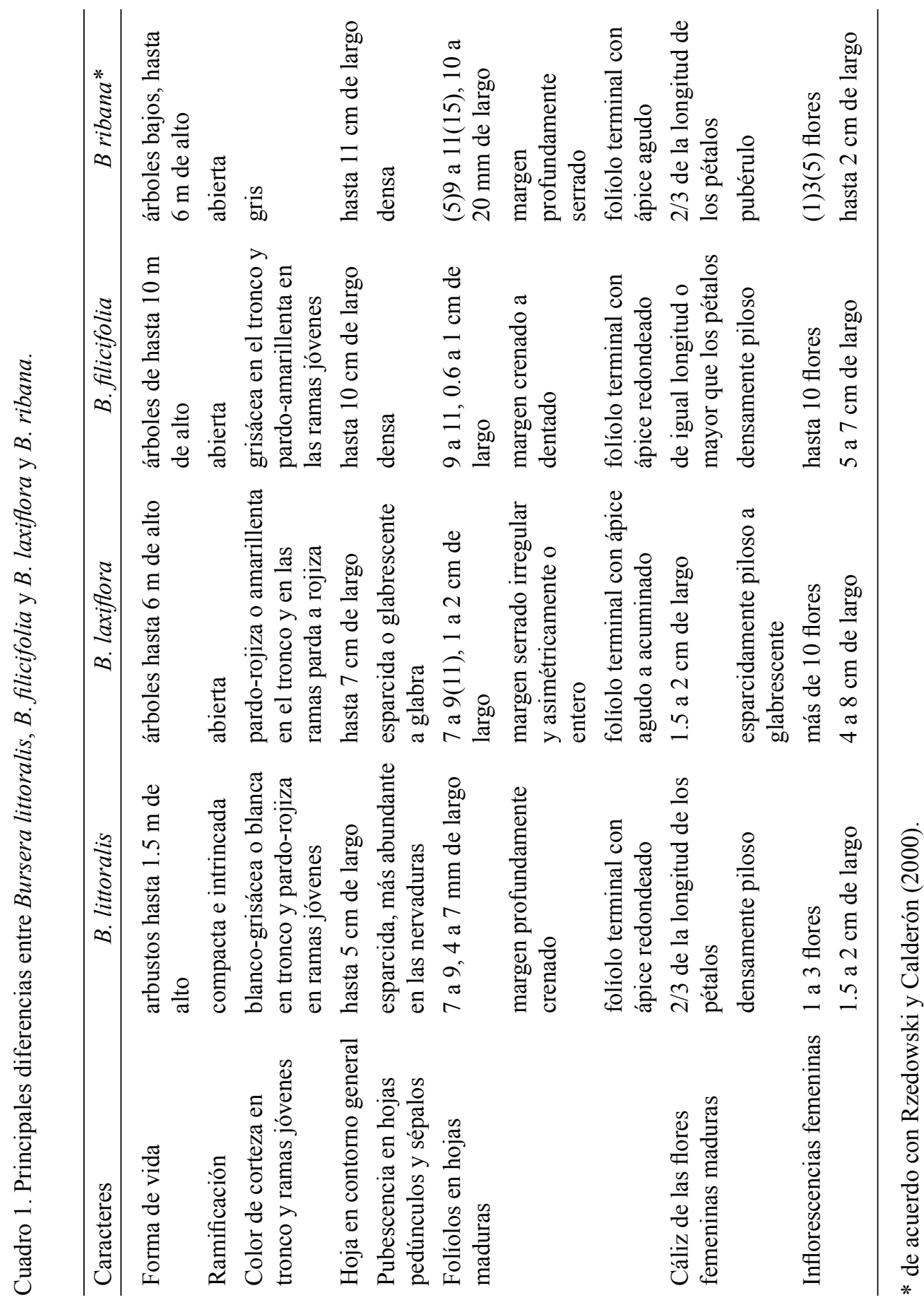


pacta, así como también por el porte bajo de la planta y el tipo de hábitat. Ambas especies no crecen juntas, aunque sus áreas respectivas son vecinas.

También, a primera vista presenta cierta relación morfológica con B. laxiflora S. Watson, sin embargo se distinguen fácilmente cuando se compara el tamaño de los folíolos, sensiblemente menores en $B$. littoralis así como por el color más claro de la corteza, tanto en el tronco como en las ramas. Además, no comparten el mismo hábitat y se distribuyen en regiones separadas. El Cuadro 1 hace referencia a la comparación de estos taxa de distribución peninsular con rasgos semejantes, también se incluye a $B$. ribana Rzed. et Calderón, de Jalisco, con la cual presenta asimismo cierta similitud.

El nombre de este nuevo taxon hace referencia a la ubicación ambiental costera.

Bursera rupicola León de la Luz sp. nov. Fig. 3.

Frutex dioica, resina aromatica. Caulis cortice brunneo-griseo non exfolianti, ramis maturis tortuosis, pendentibus; cataphylla inconspicua. Folia 1-foliolata, raro 3-foliolata, in apice brachyblastorum conglomerata, petiolata; lamina nitida, ovata, primum visum glabra sed dense pubescens trichomatibus simplicibus et trichomatibus glandularibus minutissimis. Inflorescentiae in paniculis laxis, pedunculatis, 2-6 floribus pedicellatis, pedicellis $4-9 \mathrm{~mm}$ longis, dense pubescentibus; bracteae elliptico-lanceolatae; bracteolae lineares, pubescentes. Flores masculi 5-meri; calyx brevilobulatus, lobulis triangularibus; petala per anthesin reflexa; stamina 10; discus nectarifer 10-lobulatus; gynoecium vestigiale manifestum. Flores feminei 4-meri; calyx lobulis triangularibus; staminodia 8; ovarium 2-loculare, stigma 2-lobato; pedunculi fructiferi pendentes. Fructus solitarii vel terni quaternique aggregati, bivalvati, leviter compressi, putamine nigro ad 2/3 longitudinis pseudoarillo scarlatino obtecto.

Arbustos hasta de $3 \mathrm{~m}$ de alto, dioicos, con resina aromática; tronco con corteza pardo-grisácea no exfoliante, ramas maduras tortuosas, intrincadas y colgantes, ramas jóvenes pardo-rojizas, glabras; catafilos inconspicuos, deltoides a lanceolados, 1 a $2 \mathrm{~mm}$ de largo, pubescentes con tricomas glandulares en el margen; hojas 1-folioladas, rara vez 3-folioladas, generalmente aglomeradas en el ápice de los braquiblastos, pecioladas, pecíolos de 5 a15 $\mathrm{mm}$ de largo, en fresco rojos, densamente pubescentes con tricomas diminutos, algunos glandulares, lámina ovada 


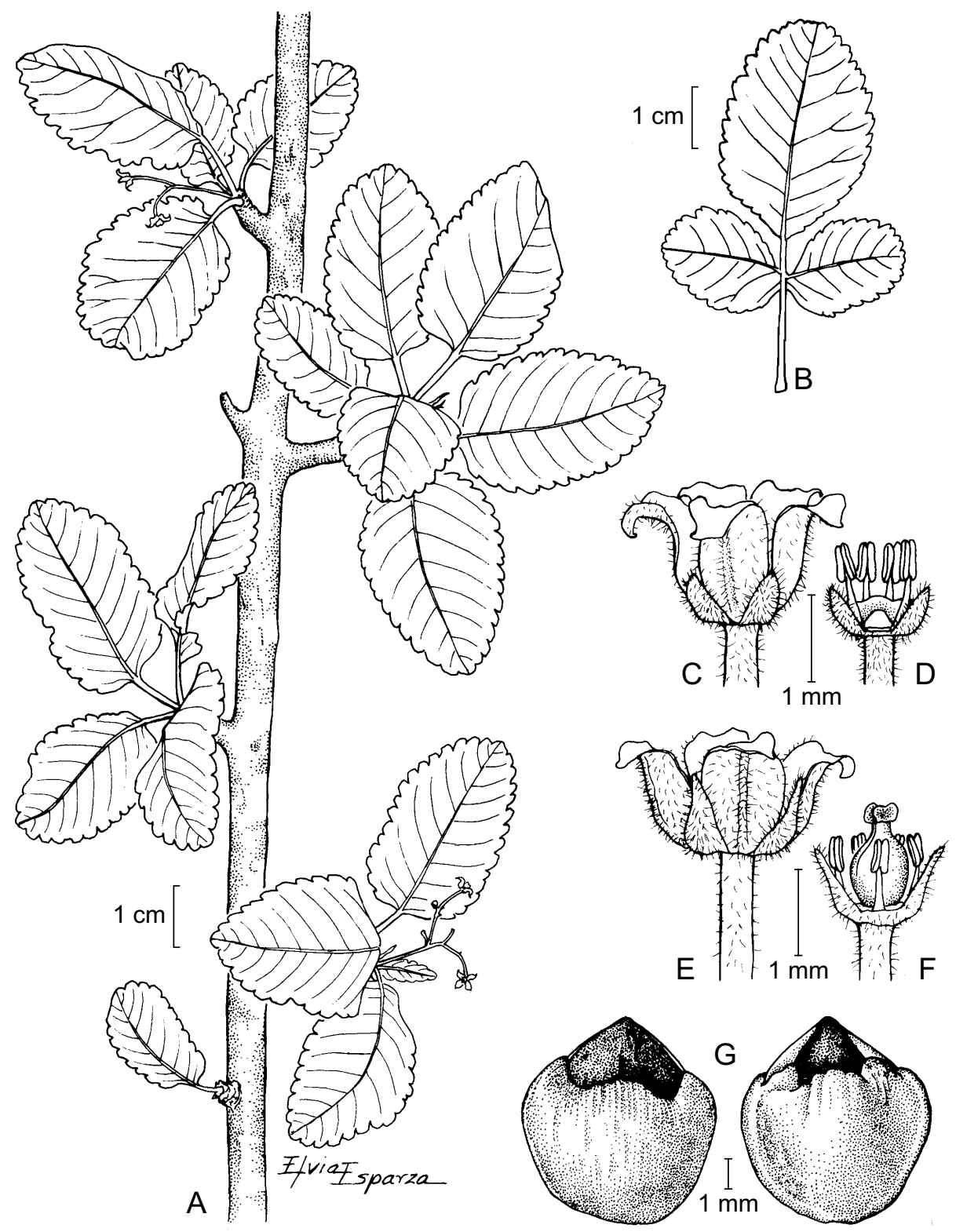

Fig. 3. Bursera rupicola Léon de la Luz. A. Rama con hojas y flores femeninas; B. Hoja trifoliolada; C. Flor masculina; D. flor desprovista de varios segmentos; E. Flor femenina; F. flor femenina desprovista de varios segmentos; G. hueso, visto por ambas superficies. Ilustrado por Elvia Esparza. 
en contorno general, de hasta $5 \mathrm{~cm}$ de largo y $3.5 \mathrm{~cm}$ de ancho, de apariencia glabra o hirsuta con tricomas glandulares diminutos, cuando 3-folioladas, los folíolos basales sésiles, folíolos elípticos a ovados, ápice generalmente obtuso, base redondeada a truncada, membranáceos, margen crenado a doblemente crenado, ciliado, haz brillante, con tricomas glandulares diminutos, abundantes en las nervaduras de ambas superficies, evidentes en la base del envés; inflorescencias en panículas laxas, pedunculadas, eje de la inflorescencia de 15 a $20 \mathrm{~mm}$ de largo, con 2 a 6 flores pediceladas, pedicelos 4 a $9 \mathrm{~mm}$ de largo, densamente pubescentes con tricomas glandulares; brácteas elíptico-lanceoladas, de 4 a $5 \mathrm{~mm}$ de largo; bractéolas lineares, de 1.5 a $2 \mathrm{~mm}$ de largo, pubescentes; flores masculinas 5-meras, cáliz con lóbulos triangulares, de 0.5 a $0.7 \mathrm{~mm}$ de largo, superficie externa densamente pilosa y glandular, superficie interna glabra o con tricomas escasos en el ápice, pétalos reflexos en antesis, blanquecino-amarillentos, de 1.7 a $2 \mathrm{~mm}$ de largo y 0.8 a $1 \mathrm{~mm}$ de ancho, lanceolados y elípticos, ápice ligeramente recurvado, con glándulas en el margen el tercio superior, estambres 10 , filamentos $0.7 \mathrm{~mm}$ de largo, anteras 0.3 $\mathrm{mm}$ de largo, disco nectarífero 10-lobulado, de $1 \mathrm{~mm}$ de diámetro, gineceo vestigial evidente; flores femeninas 4-meras, cáliz con lóbulos triangulares, de 0.5 a 0.9 $\mathrm{mm}$ de largo, superficie externa pilosa, interna glabra o con pocos tricomas en el ápice, estaminodios 8, filamentos de $7 \mathrm{~mm}$ de largo, anteras hasta $2 \mathrm{~mm}$ de largo, ovario 2-locular, estigma bilobado, $0.1 \mathrm{~mm}$ largo y $0.2 \mathrm{~mm}$ de grosor; pedúnculo fructífero colgante, de 1.5 a $2 \mathrm{~mm}$ de $\mathrm{cm}$ largo, frutos solitarios o en grupos de 3-4, ovoides, 2-valvados, ligeramente comprimidos, no apiculados, de 0.9 a $1.2 \mathrm{~cm}$ de diámetro, hueso negro, cubierto hasta $2 / 3$ de su largo por un pseudoarilo rojo escarlata.

Tipo: México, Baja California Sur: La Paz, arroyo Rancho El León, Sierra Cacachilas, J.L. León de la Luz y R. Medina Lemos 08-135 (holotipo: HCIB; isotipos: IEB, MEXU).

Material adicional examinado: México, Baja California Sur: Sierra Cacachilas, Rancho Arroyo del León, municipio La Paz, J.L. León de la Luz 2663 (HCIB), 7610 (HCIB), 9183 (HCIB); arroyo cerca de la carretera La Paz - San Juan de Los Planes, J.J. Pérez Navarro 1269 (HCIB); arroyo al sureste de La Paz, municipio La Paz, J.J. Pérez Navarro 1353 (HCIB).

Bursera rupicola constituye un endemismo adicional más para la flora de la región de Los Cabos, en Baja California Sur. La población parece ser única, 
compuesta apenas por unas decenas de individuos, en un ambiente similar al del bosque tropical caducifolio pero sin todos sus componentes locales característicos, en una elevación de $450 \mathrm{~m}$. El microambiente parece ser determinante de la existencia de la población, pues típicamente la planta se desarrolla sobre paredes de rocas graníticas, en posición colgante; aparentemente prefiere sitios de incidencia de sombra (Fig. 4), aunque algunos ejemplares se desarrollan sobre suelo arenoso y adquieren un porte arbóreo bajo. Florece y fructifica en respuesta a las lluvias del período julio-septiembre. Curiosamente, comparte el hábitat con la representante de un interesante género monoespecífico de la familia de las rubiáceas, Carterella alexanderae Terrell, especie única del género.

Este taxon es sin duda afín a B. epinnata (Rose) Engl., el copal más común en las comunidades de la región de Los Cabos, sin embargo se le distingue por su

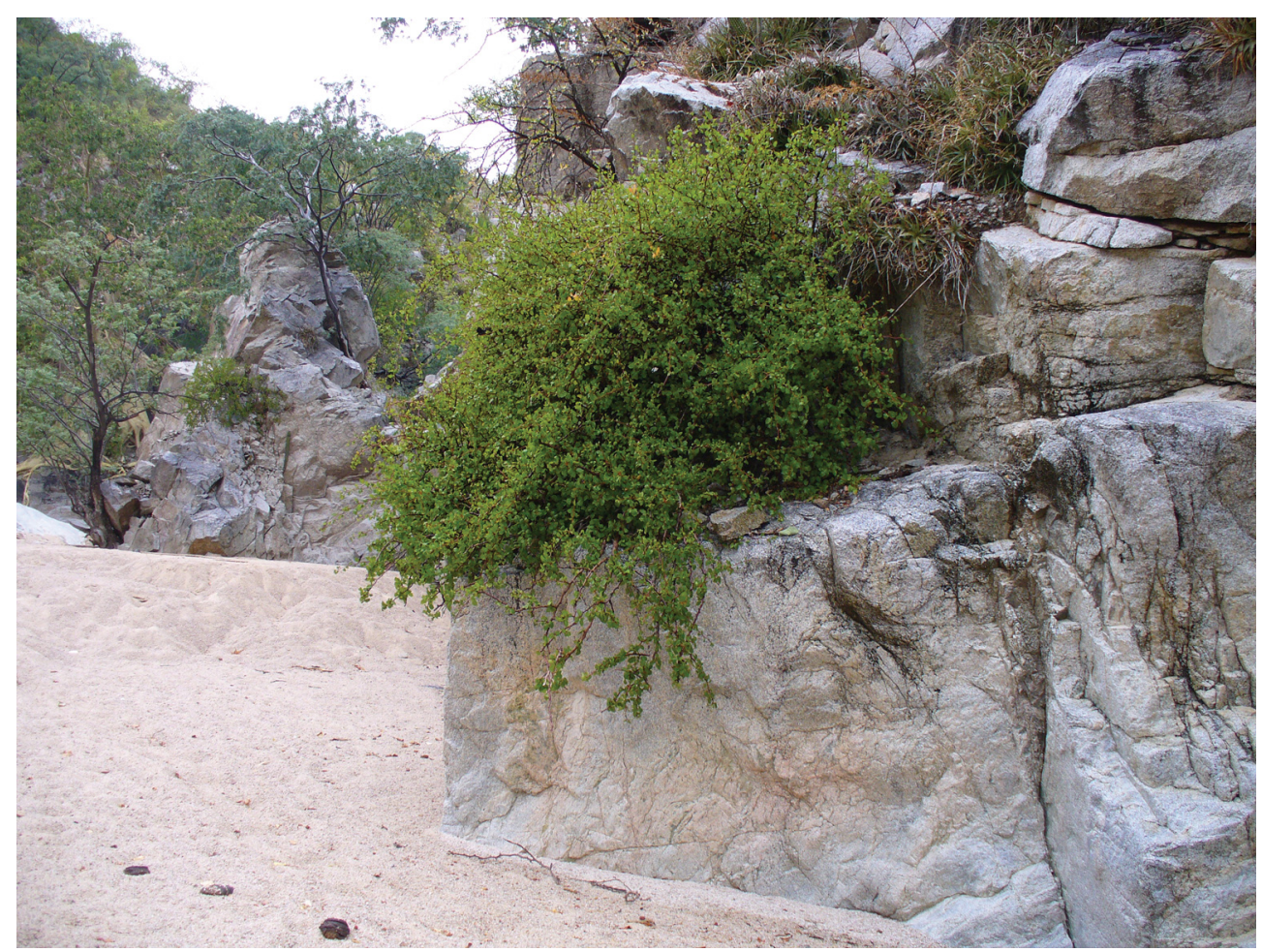

Fig. 4. Ejemplar de B. rupicola en su habitat. 
hábito arbustivo de ramificación colgante mientras que $B$. epinnata es un árbol pequeño erecto. $B$. rupicola tiene las hojas principalmente 1-folioladas y brillantes, mientras que $B$. epinnata tiene también la mayor parte de las hojas 1-folioladas, pero es común observar las 3-5-folioladas en la misma rama. El Cuadro 2 señala algunas diferencias importantes entre ambas especies. El nombre asignado a este nuevo taxon alude al sustrato del hábitat en el que se encontró, una cañada honda de paredes rocosas.

Cuadro 2. Principales diferencias entre Bursera rupicola y B. epinnata.

\begin{tabular}{|c|c|c|}
\hline Caracteres distintivos & B. rupicola & B. epinnata \\
\hline Hábito & $\begin{array}{l}\text { arbusto de hasta } 3 \mathrm{~m} \text { alto, } \\
\text { tronco corto bien definido, } \\
\text { ramas colgantes; crece sobre } \\
\text { laderas de fuerte pendiente y } \\
\text { sustrato rocoso }\end{array}$ & $\begin{array}{l}\text { árbol hasta } 7 \mathrm{~m} \text { alto, base del } \\
\text { tronco hasta } 1.5 \mathrm{~m} \text { de largo, } \\
\text { ramas erguidas, crece en los } \\
\text { valles y laderas con pendiente } \\
\text { ligera y sustrato arenoso }\end{array}$ \\
\hline Hojas maduras & $\begin{array}{l}\text { 1(3) folíolos, brillantes, } \\
\text { de apariencia glabra, con } \\
\text { tricomas simples y glandulares } \\
\text { diminutos }\end{array}$ & $\begin{array}{l}\text { 1(3-5) folíolos, opacas, } \\
\text { densamente pubescentes en } \\
\text { ambas superficies, con tricomas } \\
\text { simples más largos }\end{array}$ \\
\hline Panículas femeninas & flores escasas & flores numerosas \\
\hline
\end{tabular}

\section{AGRADECIMIENTOS}

Agradecemos al Dr. Fernando Chiang Cabrera (MEXU), la transcripción al latín de la descripción, a Elvia Esparza la elaboración de las ilustraciones y muy especialmente a Rosalinda Medina Lemos (MEXU) por su gestión, impulso y desinteresado apoyo en la culminación del manuscrito. Miguel Domínguez y Raymundo Domínguez (HCIB) participaron activamente en el trabajo de campo.

\section{LITERATURA CITADA}

Johnson, M. B. 1992. The genus Bursera (Burseraceae) in Sonora, México and Arizona, U.S.A. Desert Plants 10(3):126-143.

Kohlmann, B. y S. Sánchez-Colón. 1984. Estudio areográfico del género Bursera Jacq. ex L. (Burseraceae) en México: una síntesis de métodos. In: Ezcurra, E., M. Equihua, 
B. Kohlmann y S. Sánchez-Colón (eds.). Métodos cuantitativos en ecología. Instituto de Ecología, A.C. México, D.F. pp. 41-125.

León de la Luz, J. L., J. J. Pérez-Navarro, M. Domínguez y R. Domínguez. 1999. Serie listados florísticos de México: Flora de la Región del Cabo de Baja California Sur, México. Vol. 18. Instituto de Biología, Universidad Nacional Autónoma de México. México, D.F. 39 pp.

Rzedowski, J. y H. Kruse. 1979. Algunas tendencias evolutivas en Bursera (Burseraceae). Taxon 28: 103-116.

Rzedowski, J. y G. Calderón de Rzedowski. 2000. Tres especies nuevas de Bursera (Burseraceae) de la región costera del occidente de México. Acta Bot. Mex. 50: 4759.

Rzedowski, J., R. Medina Lemos y G. Calderón de Rzedowski. 2005. Inventario del conocimiento taxonómico, así como de la diversidad y del endemismo regionales de las especies mexicanas de Bursera (Burseraceae). Acta Bot. Mex. 70: 85-111.

Shreve, F. y I. L. Wiggins. 1964. Vegetation and flora of the Sonoran Desert. 2 vols. Stanford University Press. Stanford, CA. 1740 pp.

Wiggins, I. L. 1980. Flora of Baja California. Stanford University Press. Stanford, CA. 1025 pp. 